\title{
Distance and Similarity Measures for Intuitionistic Hesitant Fuzzy Sets
}

\author{
Xiuming Chen ${ }^{1,2 *}$, Jingming $\mathrm{Li}^{1,2}$, Li Qian ${ }^{1}$ and Xiande $\mathrm{Hu}^{1}$ \\ ${ }^{1}$ School of Information Engineering, Anhui Xinhua University, Hefei 230088, P. R. China \\ ${ }^{2}$ School of Management, Hefei University of Technology, Hefei 230001, P. R. China \\ ${ }^{*}$ Corresponding author
}

\begin{abstract}
As generalize of the hesitant fuzzy sets, intuitionistic hesitant fuzzy sets (IHFSs), which permits a memberships degree and a non-membership degree of an element to a given set, can be considered as a useful tool to express uncertain information in the human decision making process. Based on the traditional distance measures, such as Euclidean distance, Hamming distance, Hausdorff distance, and other generalized distance measures, in this paper, a variety of distance measures for intuitionistic hesitant fuzzy sets are proposed, based on which the corresponding similarity measures can be obtained. We investigate the connections of the aforementioned distance measures and further develop a number of intuitionistic hesitant ordered weighted distance measures. They can alleviate the influence of unduly large (or small) deviations on the aggregation results by assigning them low (or high) weights. A numerical example is provided to illustrate these distance and similarity measures.
\end{abstract}

Keywords- intuitionistic hesitant fuzzy sets; distance measures; similarity measures; multiple attribute decision making

\section{INTRODUCTION}

The concept of intuitionistic fuzzy set (IFS) was introduced by Atanassov [1] to generalize the concept of Zadeh's fuzzy set [2]. Each element in an IFS is expressed by an ordered pair, and each ordered pair is characterized by a membership degree and a non-membership degree. The sum of the membership degree and the non-membership degree of each ordered pair is less than or equal to one. Since it was first introduced in 1986, the IFS theory has been widely investigated and applied to a variety of fields e.g. mathematical programming,[3] decision making,[4-6] pattern recognition,[7-9] image processing, [10] grey relational analysis, [6] etc.

Hesitant fuzzy sets (HFSs), an extension of traditional fuzzy sets, can address these situations. HFSs were first introduced by Torra and Narukawa [11], and they permit the membership degrees of an element to be a set of several possible values between zero and one. HFSs are highly useful in resolving situations where people hesitate when providing their preferences in the decision-making process, and they have been a subject of great interest to researchers. Recently, Rodríguez et al. [12] presented an overview and discussed future trends for HFSs.

However, in the process of some practical group recommendation, sometimes, due to the time pressure and lack of knowledge or data or the recommenders' limited attention and information processing capacities, the recommenders cannot provide their recommendations with a single numerical value, a margin of error, some possibility distribution on the possible values, several possible numerical values, several possible interval numbers, but several possible intuitionistic fuzzy numbers. For example, to get a reasonable recommendation result, a recommendation organization, which contains a lot of recommenders, is required to estimate the degree that an item satisfies an attribute. Suppose there is a case: some recommenders provide $\{(0.7,0.2)\}$, and the others provide $\{(0.6,0.3)\}$, and these two parts cannot persuade each other to change their opinions. We can easily see that such case cannot be dealt with by fuzzy sets, hesitant fuzzy sets, and their extensions, such as interval-valued fuzzy sets, intuitionistic fuzzy sets, interval-valued intuitionistic fuzzy sets, type 2 fuzzy sets, interval-valued hesitant fuzzy sets [13]. Thus, it is very necessary to introduce a new extension of hesitant fuzzy sets to address this issue. The aim of this paper is to present the notion of intuitionistic hesitant fuzzy set (IFS), which extends the hesitant fuzzy set to intuitionistic fuzzy environments and permits the membership of an element to be a set of several possible intuitionistic fuzzy numbers. Thus, intuitionistic hesitant fuzzy set is a very useful tool to deal with the situations in which the recommenders hesitate between several possible intuitionistic fuzzy numbers to assess the degree to which an item satisfies an attribute. In the previous example, the degree to which the alternative satisfies the attribute can be represented by an intuitionistic hesitant fuzzy set $\{(0.7,0.2)$, $(0.6,0.3)\}$.

A growing number of studies focus on the distance measure and the similarity measure for HFSs [14] and some extensions of HFS [15,16]. Distance measures are fundamentally important in various fields such as decision making[17-18], risk investment [19], and pattern recognition[20].

The aforementioned measures, however, cannot be used to deal with the distance measures and similarity measures between intuitionistic hesitant fuzzy sets. Due to the fact that hesitancy is a very common problem in human decision making process as mentioned earlier, it is necessary to develop some distance measures and similarity measures for intuitionistic hesitant fuzzy sets. In order to do so, the remainder of this paper is set out as follows. In the next section, we introduce some basic concepts related to hesitant fuzzy sets and intuitionistic hesitant fuzzy sets. In Section 3, we propose some distance measures and similarity measures for intuitionistic hesitant fuzzy sets. In Section 4, an illustrative 
example is pointed out. In Section 5, concludes the paper with some remarks.

\section{PRELIMINARIES}

\section{A. Intuitionistic Fuzzy Sets}

Definition 1 ([1]) Let $X$ be a universe of discourse, an IFS $A$ in $X$ is defined as:

$$
A=\left\{<x, u_{A}(x), v_{A}(x)>\mid x \in X\right\},
$$

where the functions $u_{\mathrm{A}}(\mathrm{x})$ and $v_{\mathrm{A}}(\mathrm{x})$ denote the degrees of membership and non-membership of the element $x \in X$ to the set $A$, respectively, with the condition: $0 \leq u_{\mathrm{A}}(\mathrm{x}) \leq 1,0 \leq v_{\mathrm{A}}(\mathrm{x}) \leq 1,0 \leq$ $u_{\mathrm{A}}(\mathrm{x})+v_{\mathrm{A}}(\mathrm{x}) \leq 1 . \alpha=\left(u_{\alpha}+v_{\alpha}\right)$ is named as an intuitionistic fuzzy value (IFV).

\section{B. Hesitant Fuzzy Sets}

Definition 2 ([11]) Let $X$ be a reference set, a hesitant fuzzy set (HFS) $A$ on $X$ is defined in terms of a function $h_{A}(x)$ when applied to $X$ returns a finite subset of $[0,1]$, i.e.,

$$
A=\left\{<x, h_{A}(x)>\mid x \in X\right\}
$$

where $h_{A}(x)$ is a set of some different values in $[0,1]$, representing the possible membership degrees of the element $x \in X$ to $A$. For convenience, we call $h_{A}(x)$ a hesitant fuzzy element (HFE) [14].

\section{DISTANCE AND SIMILARITY MEASURES FOR INTUITIONISTIC HESITANT FUZZY SETS}

It has been known that in the group recommendation systems, it is somewhat difficult for the recommenders to assign exact value for a membership degree and a nonmembership degree of certain element to A, but maybe hesitant from one intuitionistic fuzzy value to another. It means that it is very necessary to introduce the concept of intuitionistic hesitant fuzzy set (IHFS).
Definition 3 Let $X$ be a fixed set; an intuitionistic hesitant fuzzy set (IHFS) on $\mathrm{X}$ is given in terms of a function that when applied to $\mathrm{X}$ returns a subset of $\mathrm{D}$. To be easily understood, we express the IHFS by a mathematical symbol, and define as:

$$
A=\left\{<x_{i}, h_{A}\left(x_{i}\right)>\mid x_{i} \in X, i=1,2, \square, n\right\}
$$

where $h_{A}\left(x_{i}\right)$ is a set of some different intuitionistic fuzzy values in $\mathrm{D}$, denoting the possible membership degree and nonmembership degree of the element $\mathrm{x} \in \mathrm{X}$ to the set $\widetilde{A}$. where

$$
h_{A_{\alpha}}\left(x_{i}\right)=\left\{\left(h_{\AA_{0}}^{\mu}\left(x_{i}\right), h_{\AA_{\alpha}}^{v}\left(x_{i}\right)\right) \mid\left(h_{\AA_{0}}^{\mu}\left(x_{i}\right), h_{\alpha_{\alpha}}^{v}\left(x_{i}\right)\right) \in h_{\AA_{\alpha}}\left(x_{i}\right)\right\}
$$

For convenience, we call $\left(h_{\AA_{0}}^{\mu}\left(x_{i}\right), h_{\alpha_{0}}^{v}\left(x_{i}\right)\right)$ an intuitionistic hesitant fuzzy element (IHFE).

It is noted that the number of values in different IHFEs may be different. To compute the distance and similarity between two IHFSs, When the length of two IHFSs are not equal, one can make them having the same number of elements through adding some elements to the IHFE which has less number of elements. In terms of the pessimistic principle, the smallest element will be added while in the opposite case, the optimistic principle may be adopted. In the present work, we use the former case. This idea has been successfully applied to distance and similarity measures for HFSs [14].

Drawing on the well-known Hamming distance and the Hausdorff distance, we present the following weighted distance measures for intuitionistic hesitant fuzzy sets.

Assume that the weight of the element $x_{i} \in X$ is $w_{i}(i=1,2, \mathrm{~L}, n)$ with $w_{i} \in[0,1]$, and $\sum_{i=1}^{n} w_{i}=1$, then we get some generalized intuitionistic hesitant fuzzy weighted distance.

Definition 4 The generalized intuitionistic hesitant fuzzy weighted normalized Hamming distance between $\mathcal{A}^{\prime}$ and $B^{\prime}$ 'are defined as follows:

$$
d_{G I H F W N H D}(A, B)=\left[\sum_{i=1}^{n} w_{i}\left(\frac{1}{2 l_{x}} \sum_{j=1}^{l_{x_{i}}}\left(\left|h_{A \sigma(j)}^{\mu}\left(x_{i}\right)-h_{B \sigma(j)}^{\mu}\left(x_{i}\right)\right|^{\lambda}+\left|h_{A \sigma(j)}^{v}\left(x_{i}\right)-h_{B \sigma(j)}^{v}\left(x_{i}\right)\right|^{\lambda}\right)\right)\right]^{\frac{1}{\lambda}}
$$

The generalized intuitionistic hesitant fuzzy weighted normalized Hausdorff distance between $\widetilde{A}_{\text {and }} \widetilde{B}_{\text {are defined }}$ as follows:

$$
d_{G I H F W N H D}^{\prime}(A, B)=\left[\sum_{i=1}^{n} w_{i}\left(\max _{j}\left(\left|h_{A \sigma(j)}^{\mu}\left(x_{i}\right)-h_{B \sigma(j)}^{\mu}\left(x_{i}\right)\right|^{\lambda},\left|h_{A \sigma(j)}^{v}\left(x_{i}\right)-h_{B \sigma(j)}^{v}\left(x_{i}\right)\right|^{\lambda}\right)\right)\right]^{\frac{1}{\lambda}}
$$


Combining the above equations, we define a generalized hybrid intuitionistic hesitant fuzzy weighted normalized

distance as follows:

$$
d_{G H I H F W N D}(A, B)=\left[\sum_{i=1}^{n} w_{i}\left(\begin{array}{l}
\frac{1}{4 l_{x}} \sum_{j=1}^{l_{x_{i}}}\left(\left|h_{A \sigma(j)}^{\mu}\left(x_{i}\right)-h_{B \sigma(j)}^{\mu}\left(x_{i}\right)\right|^{\lambda}+\left|h_{A \sigma(j)}^{v}\left(x_{i}\right)-h_{B \sigma(j)}^{v}\left(x_{i}\right)\right|^{\lambda}\right) \\
+\frac{1}{2} \max _{j}\left(\left|h_{A \sigma(j)}^{\mu}\left(x_{i}\right)-h_{B \sigma(j)}^{\mu}\left(x_{i}\right)\right|^{\lambda},\left|h_{A \sigma(j)}^{v}\left(x_{i}\right)-h_{B \sigma(j)}^{v}\left(x_{i}\right)\right|^{\lambda}\right)
\end{array}\right)\right]^{\frac{1}{\lambda}}
$$

Based on the relationship between similarity measures and distances measures, the weighted similarity measures of intuitionistic hesitant fuzzy set are defined as follows:

Definition 5 The generalized intuitionistic hesitant fuzzy weighted normalized Hamming similarity measure between $\widetilde{A}_{\text {and }} \widetilde{B}$ are defined as follows:

$$
S_{\text {GIHFWNHSM }}(A, B)=1-d_{G I H F W N H D}
$$

The generalized intuitionistic hesitant fuzzy weighted normalized Hausdorff similarity measure between $\widetilde{A}$ and $\widetilde{B}$ are defined as follows:

$$
s_{G I H F W N S M}^{\prime}(A, B)=1-d_{G I H F W N H D}^{\prime}(A, B)
$$

Combining the above equations, we define a generalized hybrid intuitionistic hesitant fuzzy weighted normalized similarity measure as follows:

$$
S_{\text {GHIHFWNSM }}(A, B)=1-d_{\text {GHIHFWND }}(A, B)
$$

\section{AN APPROACH BASED ON SIMILARITY MEASURES TO MULTI-CRITERIA DECISION MAKING WITH IHFSS}

Multi-criteria decision making, which can be characterized in terms of a process of choosing or selecting sufficiently good alternative(s) (or course(s)) from a set of alternatives to attain a goal (or goals), often happens in our daily life. For example, choosing a car to buy, or selecting an electronic product from amazon or ebay. A multi-criteria decision making problem with IHFS information can be interpreted as follows: Suppose that a decision maker is asked to evaluate a set of alternatives $X=\left\{X_{1}, x_{2}, \ldots, X_{n}\right\} \quad$ with respect to several criteria $c_{j}(j=1,2, \mathrm{~L}, m)$. The criteria have a weighting vector $w=\left\{w_{1}, w_{2}, \ldots, w_{m}\right\}$, where $0 \leq w_{j} \leq 1$ and $\sum_{\mathrm{j}=1}^{\mathrm{m}} w_{j}=1$. The decision maker might feel much easier and are more willing to give their assessments by providing some IHFS information.

Example. Consider a movie recommendation system. To better recommend different types of movies on the market, we calculate their distance and similarity according to four attributes: story $\left(x_{1}\right)$, acting $\left(x_{2}\right)$, visuals $\left(x_{3}\right)$ and direction $\left(x_{4}\right)$. The weighing vector of these four attributes is $w=(0.35,0.3,0.2$, $0.15)$. Suppose that a group intends to give ratings on five movies $M_{1}, M_{2}, M_{3}, M_{4}$. Given the recommenders who make such a recommendation have different backgrounds and levels of knowledge, interests and hobbies, skills, experience and personality, etc., this could lead to a difference in the recommendation information. To clearly reflect the differences of the opinions of different recommenders, the data of recommendation information are represented by the IHFSs and listed in Table 1.

TABLE I. THE DATA OF RECOMMENDATION INFORMATION

\begin{tabular}{lllll}
\hline & $x_{1}$ & $x_{2}$ & $x_{3}$ & $x_{4}$ \\
\hline$M_{1}$ & $\{(0.5,0.3),(0.4,0.2)\}$ & $\{(0.5,0.3)\}$ & $\{(0.6,0.2)\}$ & $\{(0.6,0.4)\}$ \\
$M_{2}$ & $\{(0.8,0.1),(0.7,0.2)\}$ & $\{(0.7,0.2),(0.6,0.3)\}$ & $\{(0.7,0.2),(0.6,0.3)\}$ & $\{(0.7,0.2)\}$ \\
$M_{3}$ & $\{(0.9,0.1),(0.8,0.1)\}$ & $\{(0.8,0.1),(0.7,0.2)\}$ & $\{(0.8,0.1)\}$ & $\{(0.9,0.1)\}$ \\
$M_{4}$ & $\{(0.3,0.6)\}$ & $\{(0.5,0.4)\}$ & $\{(0.6,0.2),(0.5,0.3)\}$ & $\{(0.3,0.6)\}$ \\
$M_{5}$ & $\{(0.5,0.3)\}$ & $\{(0.2,0.6)\}$ & $\{(0.3,0.5),(0.2,0.6)\}$ & $\{(0.1,0.8)\}$ \\
\hline
\end{tabular}

Therefore, we define each ideal IHFE $h_{i j}^{*}=(1,0),(j=1,2, \mathrm{~L}, n) \quad$ in the ideal item $M=\left\{<x_{j}, h_{j}^{*}>\mid x_{j} \in X\right\},(j=1,2, \mathrm{~L}, n)$. We use the generalized intuitionistic hesitant fuzzy weighted normalized Hamming similarity measure, the generalized intuitionistic hesitant normalized Hausdorff similarity measure, and the generalized hybrid intuitionistic hesitant weighted normalized similarity measure to calculate the deviations between each alternative and the ideal alternative, then we get the rankings of these alternatives, which are listed in Figure 1-3, respectively, and variation of similarity measure values with respect to the parameter $\lambda$. We can rank the movies in accordance with the generalized intuitionistic hesitant fuzzy weighted normalized 
Hamming similarity measure: $M_{3}>M_{2}>M_{1}>M_{4}>M_{5}$, The movie $M_{3}$ is the best choice according to this similarity measure. Using the generalized intuitionistic hesitant normalized Hausdorff similarity measure, or the generalized hybrid intuitionistic hesitant weighted normalized similarity measure, we can get the same conclusion.

We find that the rankings are different as the parameter $\lambda$ (which can be considered as the decision makers' risk attitude) changes, consequently, the proposed distance measures can provide the decision makers more choices as the different values of the parameter are given according to the decision makers' risk attitudes.

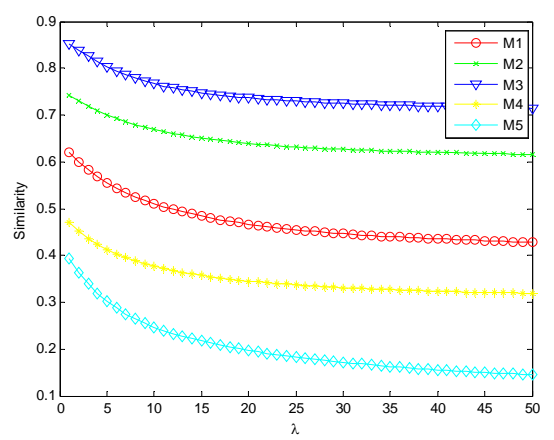

FIGURE I. VARIATION OF THE $\boldsymbol{S}_{\text {GIHFOWNSM }}$ WITH RESPECT TO THE PARAMETER $\lambda$

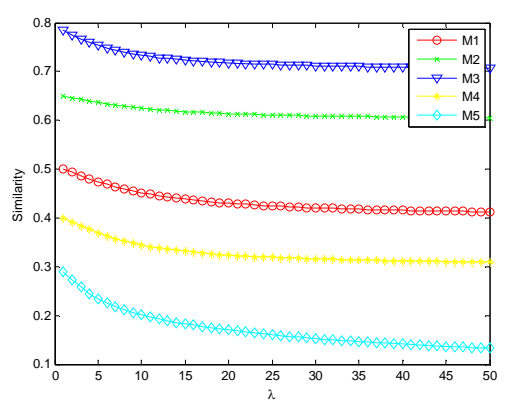

FIGURE II. VARIATION OF THE $s_{\text {GIHFOWNHSM }}^{\prime}$ WITH RESPECT TO THE PARAMETER $\lambda$

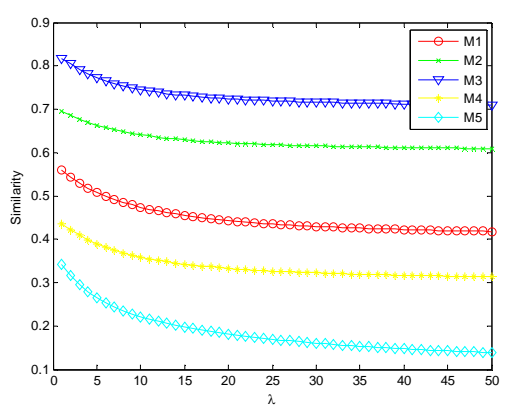

FIGURE III. VARIATION OF THE $s_{\text {GHIHFOWNSM }}$ WITH RESPECT TO THE PARAMETER $\lambda$

\section{CONCLUSION}

As generalize of the hesitant fuzzy sets, intuitionistic hesitant fuzzy sets, which permits the memberships of an element to a given set having a few different intuitionistic fuzzy sets rather than real numbers, can be considered as a powerful tool to express uncertain information in the human decision making process. Based on the traditional Hamming distance, Euclidean distance, Hausdorff distance, and generalized distance, in this paper, we propose a variety of distance measures for intuitionistic hesitant fuzzy sets, based on which the corresponding similarity measures can be obtained. We investigate the connections of the aforementioned distance measures. They can alleviate the influence of unduly large (or small) deviations on the aggregation results by assigning them low (or high) weights. Finally, we shall present a numerical example to show potential evaluation of emerging technology commercialization with intuitionistic hesitant fuzzy information in order to illustrate the method proposed in this paper. In the future, we shall continue working in the application of the intuitionistic hesitant fuzzy multiple attribute decision making to other domains.

\section{ACKNOWLEDGMENT}

The authors would like to thank the anonymous reviewers for their valuable suggestions as to how to improve this paper. This work was supported by the Natural Science Foundation of the Anhui Educational Committee of China (Grant Nos. KJ2015A300, KJ2014A100).

\section{REFERENCES}

[1] K. T. Atanassov, Intuitionistic fuzzy sets, Fuzzy Sets Syst. 20(1) (1986) $87-96$.

[2] L.A. Zadeh, Fuzzy Sets Inf. Control 8 (1965) 338-353.

[3] D. F. Li, Mathematical-programming approach to matrix games with payoffs represented by Atanassov's interval-valued intuitionistic fuzzy sets, IEEE Trans. Fuzzy Syst. 18(6) (2010) 1112-1128.

[4] G.W. Wei, X.F. Zhao, Some induced correlated aggregating operators with intuitionistic fuzzy information and their application to multiple attribute group decision making, Expert Syst. Appl. 39 (2) (2012) 20262034.

[5] Z.L. Yue, Y.Y. Jia, G.D. Ye, An approach for multiple attribute group decision making based on intuitionistic fuzzy information, Int. J. Uncertainty Fuzziness Knowl. Based Syst. 17 (3) (2009) 317-332.

[6] G.W. Wei, Gray relational analysis method for intuitionistic fuzzy multiple attribute decision making, Expert Syst. Appl. 38 (9) (2011) 11671-11677.

[7] D.F. Li, C.T. Cheng, New similarity measures of intuitionistic fuzzy sets and application to pattern recognitions, Pattern Recognit. Lett. 23 (2002) 221-225.

[8] S.K. De, R. Biswas, A.R. Roy, An application of intuitionistic fuzzy sets in medical diagnosis, Fuzzy Sets and Systems 117 (2001) 209-213.

[9] I.K. Vlachos, G.D. Sergiadis, Intuitionistic fuzzy information applications to pattern recognition, Pattern Recognition Letters 28 (2007) 197-206.

[10] T. Chaira, Intuitionistic fuzzy segmentation of medical images, IEEE Trans. Biomed. Eng. 7(6) (2010) 1430-1436.

[11] V. Torra, Hesitant fuzzy sets, Int. J. Intell. Syst. 25 (2010) 529-539.

[12] R.M. Rodríguez, L. Martínez, V. Torra, Z.S. Xu, F. Herrera, Hesitant fuzzy sets: state of the art and future directions, Int. J. Intell. Syst. 29 (2014) 495-524. 
[13] N. Chen, Z.S. Xu, M.M. Xia, Interval-valued hesitant preference relations and their applications to group decision making, Knowl.-Based Syst. 37 (2013) 528-540.

[14] Z. Xu, M. Xia, Distance and similarity measures for hesitant fuzzy sets, Information Sciences 181 (2011) 2128-2138.

[15] B. Farhadinia, A novel method of ranking hesitant fuzzy values for multiple attribute decision-making problems, International Journal of Intelligent Systems (2013) 1-16. doi:10.1002/int.21600.

[16] B. Farhadinia, Information measures for hesitant fuzzy sets and intervalvalued hesitant fuzzy sets, Information Sciences 240 (2013) 129-144.

[17] D.H. Peng, Ch.Y. Gao, Zh.F. Gao, Generalized hesitant fuzzy synergetic weighted distance measures and their application to multiple criteria decision making, Applied Mathematical Modelling 37 (2013) 58375850 .

[18] N. Chen, Z.S. Xu, M.M. Xia, Interval-valued hesitant preference relations and their applications to group decision making, KnowledgeBased Systems 37 (2013), 528-540.

[19] X. Gu, Y. Wang, B. Yang, A Method for Hesitant Fuzzy Multiple Attribute Decision Making and Its Application to Risk Investment 6(6) (2011), 282-287.

[20] Z.S. Xu, M. Xia, On distance and correlation measures of hesitant fuzzy information, International Journal of Intelligence Systems 26(5) (2011), 410-425.

M.M. Xia, Z.S. Xu, Hesitant fuzzy information aggregation in decision making, Int. J. Approx. Reason. 52 (2011) 395-407. 